\title{
Error Estimation in Recording $I-V$ Characteristics of Josephson Junctions
}

\author{
S. I. Borovitskiŭ, V. D. Gelikonova, A. V. Komkov, Kh. A. Aŭnitdinov ${ }^{\dagger}$, and A. M. Klushin \\ Kvarts Institute of Electronic Measurements, Nizhni Novgorod, 603009 Russia \\ e-mail: cel@sandy.ru \\ Received February 20, 2001; in final form, February 11, 2002
}

\begin{abstract}
A method for characterizing a current total step in the $I-V$ characteristic of a Josephson junction array is considered. In this method, an appropriately selected approximating curve is statistically fitted to the experimentally found curve. A self-calibration algorithm for an array of junctions incorporated into programmable voltage standards is suggested. (C) 2002 MAIK "Nauka/Interperiodica".
\end{abstract}

\section{INTRODUCTION}

In recent years, interest has arisen in programmable voltage standards based on Josephson junction arrays [1]. With such standards, it becomes possible to produce a set of quantized reference voltages from 0 to $10 \mathrm{~V}$ and simplify the calibration of precision ADCs and digital voltmeters. Accordingly, the calibration scheme for these devices radically changes, and the certification speed and accuracy are greatly improved. To produce a set of precision reference voltages, an array of a large number $m$ of series-connected nonhysteresis Josephson junctions is used. One of the working algorithms for programmable voltage standards uses the quantized reference voltage across a junction array at the first step with index $n= \pm 1$. The total voltage is then $V_{J}=\left(m_{1}-m_{2}\right) f / K_{J}[2]$. Here, $m_{1}$ is the number of junctions at the step $n=1, m_{2}$ is that at the step $n=-1, f$ is the frequency of an applied harmonic signal, and $K_{J}=$ $483.5979 \mathrm{GHz} / \mathrm{V}$ is the Josephson constant. It should be emphasized that this algorithm allows for the application of Josephson junctions on high-temperature superconductors with a great $(\approx 100 \%)$ spread in Josephson critical currents $\delta I=I_{\mathrm{c}, \max } / I_{\mathrm{c}, \min }$, where $I_{\mathrm{c}, \max }$ and $I_{\mathrm{c}, \min }$ are, respectively, the maximal and minimal critical currents of the junctions incorporated into the array [3,4]. At $f \geq K_{J} I_{\mathrm{c}, \max } R_{\mathrm{N}}\left(R_{\mathrm{N}}\right.$ is the normal resistance of the junctions), the microwave signal power can be selected such that the amplitude of the first total current step $\Delta I_{1}$ is on the order of $I_{\mathrm{c} \text {, min }}$. Actually, $\Delta I_{1}$ decreases because of a spread in $R_{\mathrm{N}}$ and the nonuniform distribution of the microwave current along the array. A recent investigation of a Josephson programmable standard based on niobium nonhysteresis junctions has shown that, after each cooling of the junctions, a new distribution of the microwave power along the array arises and the system must be tuned to the optimal frequency of irradiation

\footnotetext{
$\dagger$ Deceased.
}

[5]. At low $I_{c, \text { min }}$ and/or elevated temperatures $(\approx 78 \mathrm{~K})$, thermal noise may additionally decrease $\Delta I_{1}$ and accordingly increase the inaccuracy of the voltage standard $[4,6]$. Because of this, the control of the slope and other parameters of the current total step is of great importance.

In this work, we suggest a possible way of characterizing current total steps appearing in the $I-V$ characteristic of a Josephson junction array and discuss a selfcalibration algorithm for the array. The feasibility of the method is demonstrated with the critical current of a bicrystal Josephson junction made of a high-temperature semiconductor.

\section{PRECISE METHOD FOR MEASURING THE CURRENT STEP PARAMETERS}

In the conventional technique for finding the current step parameters (specifically, its slope), the voltage $V_{J}$ of the step is successively measured at several points $(i \approx 10)$ the number of which depends on the constant bias current passing through the array. To improve the measurement accuracy, the voltage to be measured first is shifted to the zero level by inserting a known voltage of opposite polarity and second is measured $N$ times at each $i$ th point [7]. The compensation of $V_{J}$ allows one to perform measurements with a minimal standard deviation $\sigma$ depending on the intrinsic noise of a nanovoltmeter. In the best digital nanovoltmeters, $\sigma \approx 10 \mathrm{nV}$; in analog devices, $\sigma \leq 1 \mathrm{nV}$. As the number of independent measurements grows, the standard deviation of the mean value decreases as $\sigma_{m}=\sigma /(N-1)^{-1 / 2}$. For the time of single-point measurement $\tau=100-1000 \mathrm{~s}$, the value of $\sigma_{m}$ can be reduced to $\leq 0.1 \mathrm{nV}$ [8]. However, small$\sigma_{m}$ measurements at all $i$ points take a considerable amount time (about $10 \tau$ ), during which the stability of the thermoelectric voltage in the measuring circuit is hard to maintain. In this case, the drift of the thermal emf will make a major contribution to the total standard 
deviation of the measurements. There are a number of methods taking into account the thermal emf drift [7]. However, their use is associated with a further increase in the number of measurements and, hence, elongates the measuring cycle.

The essence of our method is as follows. First, for a time $\approx \tau$, the $I-V$ characteristic $V_{k}=f\left(I_{k}\right)$ is recorded in the range of a step of the current (or critical current) being studied, where the subscript $k$ runs from 1 to $N$. Then, the data array obtained is approximated by the mathematical expression $V=f\left(I, p_{1}, p_{2}, p_{3}, \ldots\right)$, where $V$ is the voltage across the junction; $I$ is the bias current; and $p_{1}, \mathrm{p}_{2}, p_{3}, \ldots$ are parameters of the theoretical model. The mean values of the parameters and their standard deviations are found by the least squares method. Thus, the problem is reduced to finding an approximating curve that adequately fits experimental data.

As a curve approximating the $I-V$ characteristic of a Josephson junction near a step of the current (or $I_{\mathrm{c}}$ ), we take the expression

$$
V=V_{0}+r\left(I-i_{0}\right)+\frac{r_{d}}{i_{1}+i_{2}}\left(i_{1}^{2} e^{\left(I-i_{0}\right) / i_{1}}-i_{2}^{2} e^{-\left(I-i_{0}\right) / i_{2}}\right) .
$$

Expression (1) involves six parameters. The parameter $V_{0}$ specifies the position of the current step on the voltage axis and tends toward $V_{J}$ in the optimum case. The factor $r$ reflects the presence of parasitics in the measuring circuit. The study of this factor can shed light on the value and nature of the parasitics and help in eliminating them or reducing to a reasonable level. Approximating curve (1) has an inflection where the current and the differential resistance equal, respectively, $i_{0}$ and $r_{\mathrm{d}}$. The parameter $i_{0}$ defines the position of the current step midpoint on the current axis. The parameters $i_{1}$ and $i_{2}$ have the dimension of current and specify the bend (smooth or sharp) of the $I-V$ curve at the edges of the steps.

When selecting the form of formula (1), we took into consideration that junctions with the nonhysteresis $I-V$ curve may exhibit one-particle tunneling of magnetic quanta at the edges of $I_{c}$, causing the exponential current dependence of the voltage, and that ranges where this takes place are much smaller than the critical current. From the theory of the Josephson effect [9], it is known that the current-voltage characteristic near the step has a similar form. However, in analyzing data for current total steps, one should bear in mind that the parameters $i_{1}$ and $i_{2}$ involved in the exponential dependence depend largely on the spread in $R_{N}$ of the junctions that are series-connected to form an array and on the nonuniformity of the microwave field along the array, rather than on the conduction mechanism. Formula (1) is a good fit to the characteristic only in the vicinity of the voltage $V_{0}$; at higher voltages, the $I-V$ curve describes resistive regions, where expression (1) is invalid.

\section{RESULTS AND DISCUSSION}

The statistical fit of the approximating curve to experimental data implies the minimization of the total standard deviation

$$
\chi=\sqrt{\frac{\sum_{k=1}^{N}\left(V_{k}-V\left(I_{k}\right)\right)^{2}}{N-p}},
$$

where $p$ is the number of desired parameters [10].

Let us consider sum (2) as a function of the number $N$ of points and the range of fit. By way of example, we will look for the parameters of curve (1), which describes the $I-V$ characteristic of a Josephson junction in the vicinity of the critical current.

We studied the $I-V$ characteristic of a bicrystal Josephson junction based on a high-temperature superconductor [3, 6]. The characteristic was recorded according to the scheme shown in Fig. 1 with the program developed in [11]. As a voltage amplifier, a nanovoltmeter with an intrinsic noise of $1-2 \mathrm{nV}$ and a gain of $5 \times 10^{4}$ was used. Such nanovoltmeters are incorporated into industrial Josephson voltage standards [12]. Figure 2 shows the record of the critical current and its approximation by function (1). The table summarizes the fitting parameters and their standard deviations corresponding to a confidence interval of $2.8 \chi$. The probability of falling outside this interval is no more than $1 \%$.

The parameter $\chi v s$. number of points $N$. It is known that the minimizing procedure is aimed at finding the absolute, rather than relative, minimum of sum (2). To find the absolute minimum, one usually changes the

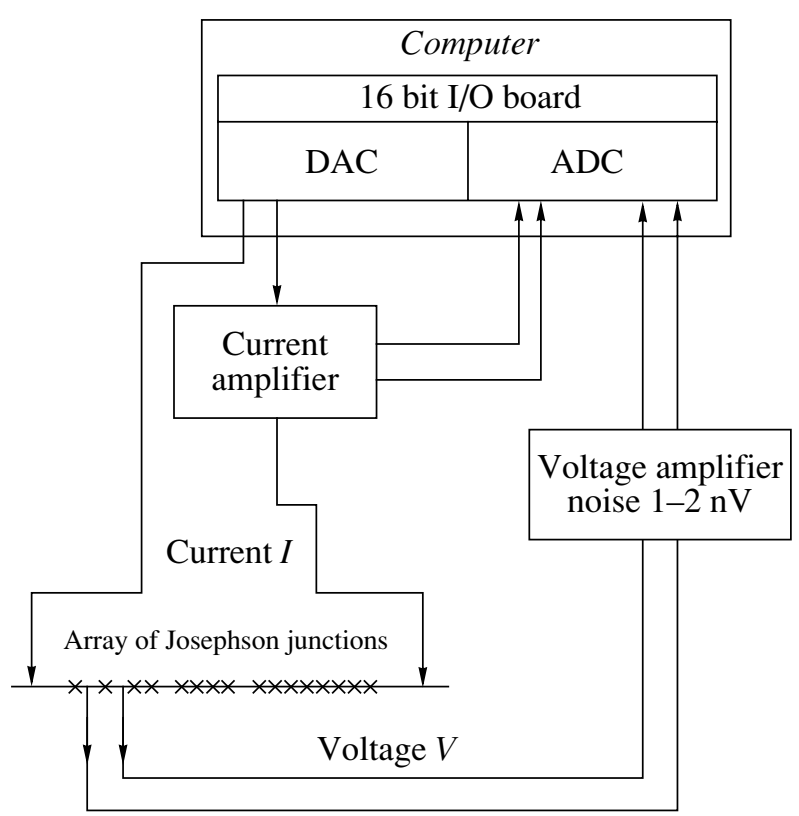

Fig. 1. Precision measurement of the current-voltage characteristic of Josephson junctions. 


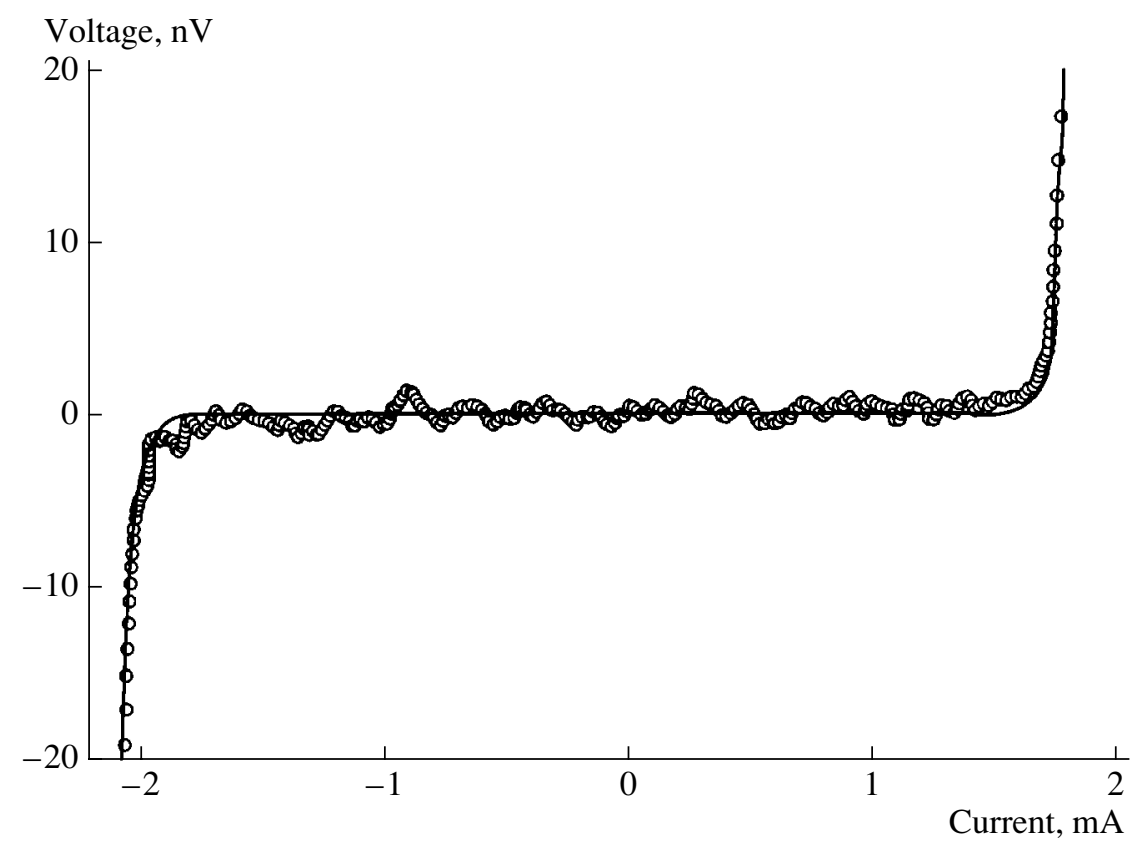

Fig. 2. $I-V$ characteristic of a bicrystal Josephson junction near the critical current: $\bigcirc$, data points; solid line, fitting curve.

initial conditions of the process and monitors the value of $\chi$ and the parameters of the fitting curve. When the final result becomes independent of the initial conditions, it can be argued that the absolute minimum is found [10]. To check this statement, we studied the dependence of the approximation parameters and their standard deviations on the number of points used for the approximation. The number of points was varied from $N=473$ to $N=3786$. Figures 3 and 4 show the values of the parameters and their standard deviations normalized to the related means. As follows from Fig. 3, the approximation parameters depend on $N$ only slightly. From Fig. 4, $\chi$ is seen to be independent of the

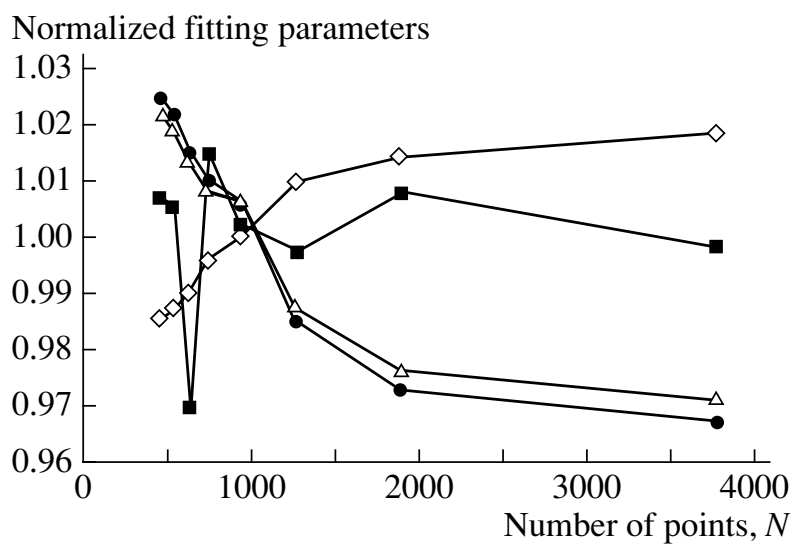

Fig. 3. $(\boldsymbol{\square}),(\diamond),(\bullet)$, and $(\triangle)$ are the normalized fitting parameters $V_{0}, i_{0}, i_{1}$, and $i_{2}$ vs. number of points $N$ taking part in the approximation. Solid lines connect corresponding symbols. number of points, whereas the normalized standard deviations of the parameters in expression (1) decrease as $N^{-1 / 2}$. These observations suggest that the absolute minimum of sum (2) is reached and the parameter means found provide the best fit to the experimental curve. Moreover, it can be argued that $N$-related systematic errors, if present, play an insignificant role.

The effect of the approximation voltage range on $\chi$. Figure 5 shows the dependence of $\chi$ on the approximation voltage range. At voltages above $20-30 \mathrm{nV}$, the total standard deviation $\chi$ grows rapidly. Figure 6 demonstrates the differences $V_{k}-V\left(I_{k}\right)$ between the $I-V$ curve recorded and the fitting curves with voltages of $\pm 9, \pm 20$, and $\pm 160 \mathrm{nV}$, along with the experimental dependence $V_{k}\left(I_{k}\right)$. It is seen that at low voltages the approximation gives $I_{c}$ higher than the values measured; at high voltages, the differences are due to the limited applicability domain of formula (1). Note also that the standard deviations of all the approximation parameters, except $\sigma_{V_{0}}$, grow near zero. From these dependences, one can conclude that the optimal range of approximation is from \pm 20 to $\pm 30 \mathrm{nV}$ in our case.

The contribution of the standard deviations of the parameters to the standard deviation of the voltage. The standard deviation of the voltage depends on the bias current as

$$
\sigma_{V}^{1}(I)=\sqrt{\sum_{i=1}^{6}\left(\frac{\partial V(I)}{\partial p_{i}}\right)^{2} \sigma p_{i}^{2}} .
$$




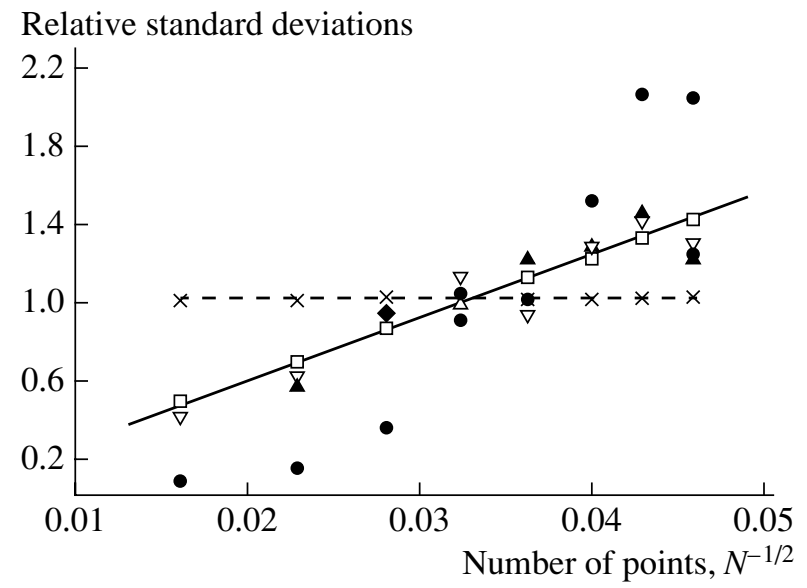

Fig. 4. Normalized standard deviations $(\square) \sigma_{V_{0}},(\bullet) \sigma_{i_{0}}$, $(\bigcirc) \sigma_{\mathrm{rd}},(\mathbf{\Lambda}) \sigma_{i_{1}},(\nabla) \sigma_{i_{2}}$ and $(\times)$ normalized total standard deviation $\chi$ vs. number of points $N$ participating in the approximation. The solid line represents the linear fit to the parameter standard deviations vs. $N$ dependence.

The contributions of the standard deviations of the parameters to $\sigma_{V}^{1}(I)$ are different. Figure 7 shows the logarithm of the contribution of the parameter standard deviations to $\sigma_{V}^{1}(I)$ vs. bias current for a voltage range from -20 to $20 \mathrm{nV}$. From the curves in Fig. 7, it follows that, at currents equal to or higher than $I_{\mathrm{c}}$, the major contribution to the total error is from $\sigma_{V_{0}}^{1}(I)$ and $\sigma_{r}^{1}(I) \approx$ $\sigma_{V_{0}}^{1}(I)$. Thus, in this current range, $\sigma_{r}^{1}(I) \approx\left(2 \sigma_{V_{0}}(I)\right)^{1.2}$ and grows rapidly when the current exceeds $I_{\mathrm{c}}$. The standard deviation $\sigma_{V_{0}}$, as well as all other standard deviations, decreases with increasing $N$. In our example (Fig. 2), it became 50 to 100 times lower than the intrinsic noise of the nanovoltmeter, not exceeding $\sigma_{V_{0}}=$ $20 \mathrm{pV}$. The low mean value of the differential resistance $r_{\mathrm{d}}$ (see table) indicates the absence of the slope of critical current and demonstrates the potentialities of the method as applied to measuring low parasitic resistances.

\section{SELF-CALIBRATION OF JUNCTION ARRAYS FOR A PROGRAMMABLE VOLTAGE STANDARD}

The method suggested allows for the natural selfcalibration of an array of $2^{m}$ junctions that is incorporated into a programmable voltage standard [2]. The array is subdivided into $(m+1)$ sections (bits). The number of junctions in the first $m$ bits corresponds to the binary code $(1,2,4,8 \ldots)$. The last, $(m+1)$, bit has only one junction. During measurements, the total step must be made coincident with the zero voltage level to retain the desired accuracy. Therefore, the calibration of the entire array is carried out in several stages. First,
Standard deviations, $\mathrm{nV}$

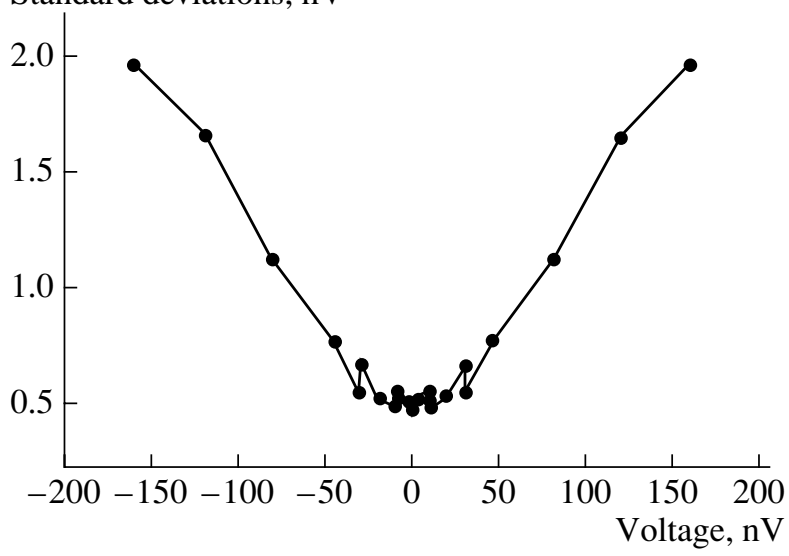

Fig. 5. Total standard deviation $\chi$ vs. fitting voltage range $(\bullet)$. The solid line connects data points.

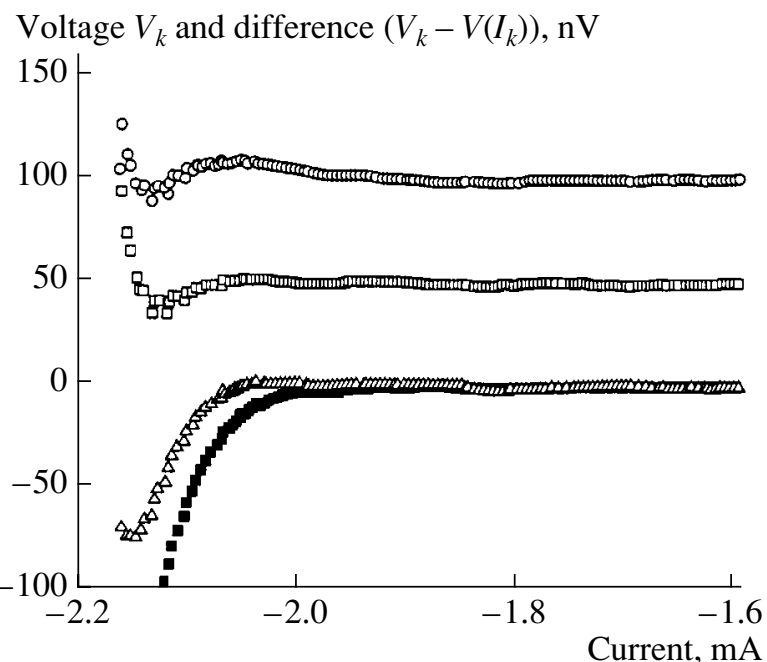

Fig. 6. Difference between the experimental $I-V$ characteristic (ם) and fitting curves for voltages in the range from $(\square)-9$ to $+9,(\triangle)-20$ to +20 , and $(\bigcirc)-160$ to $+160 \mathrm{nV}$. For convenience, the differences calculated for the ranges \pm 9 and $\pm 160 \mathrm{nV}$ are shifted by 50 and $100 \mathrm{nV}$ relative to the zero level.

the voltage of the step +1 of one junction is compared with that of the step -1 of the second junction. Then, these two junctions are connected in series and their

Fitting parameters and their standard deviations

\begin{tabular}{c|c|c|c}
\hline Parameters & $\begin{array}{c}\text { Units of } \\
\text { measure }\end{array}$ & $\begin{array}{c}\text { Parameter } \\
\text { values }\end{array}$ & $\begin{array}{c}\text { Standard } \\
\text { deviations }\end{array}$ \\
\hline$V_{0}$ & $\mathrm{nV}$ & 0.13 & 0.02 \\
$r$ & $\Omega$ & $2.9 \times 10^{-7}$ & $2 \times 10^{-8}$ \\
$i_{0}$ & $\mathrm{~mA}$ & 0.08 & 0.05 \\
$r_{\mathrm{d}}$ & $\Omega$ & $7.6 \times 10^{-21}$ & $8.5 \times 10^{-21}$ \\
$i_{1}$ & $\mathrm{~mA}$ & 0.04 & 0.002 \\
$i_{2}$ & $\mathrm{~mA}$ & 0.05 & 0.002 \\
$\chi$ & $\mathrm{nV}$ & 0.41 & \\
\hline
\end{tabular}


Standard deviations of the fitting parameters, logarithmic scale

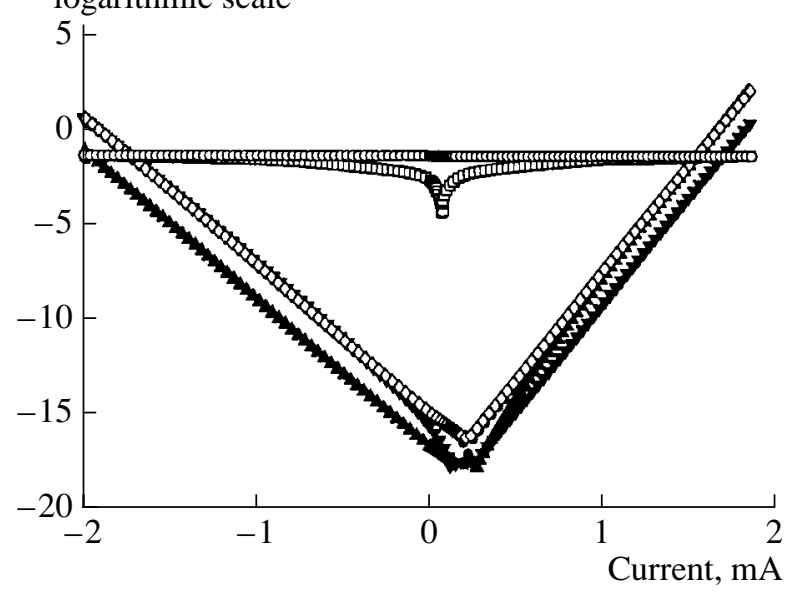

Fig. 7. Logarithmic contribution of the parameter standard deviations vs. bias current for voltages in the range $\pm 20 \mathrm{nV}$.
(○) $\log \sigma_{V_{0}}^{1},(\square) \log \sigma_{r}^{1},(\diamond) \log \sigma_{i_{0}}^{1}, \quad(\boldsymbol{\nabla}) \log \sigma_{r d}^{1}$,
(ム) $\log \sigma_{i_{1}}^{1}$, and $(\bullet) \log \sigma_{i_{2}}^{1}$.

total voltage is compared with that of a section of two junctions that have opposite polarity, etc. Having established (with a high accuracy) the absence of the slope on the first steps of individual contacts, one can establish the absence of parasitic slopes on total steps in all the bits of the array. This algorithm allows one to automate the self-calibration of arrays of series-connected junctions.

\section{CONCLUSION}

We suggest a method for processing a body of experimental data for the $I-V$ characteristics of Josephson junctions that can estimate the accuracy of measurements. For this purpose, it is necessary to select an analytical expression for the $I-V$ characteristic that involves a small number of parameters. The choice can be based on both a theoretical model and intuitive concepts.
The desired mean values of the parameters and their standard deviations are found by the least squares method. This method combines the advantage of reducing the problem to finding a function depending on a small number of parameters and the advantages of a large number of measurements. A small value of $\chi$ found by fitting is the best corroboration of the potentialities of the method. Another verification of the method is the study of the dependence of the parameters on the number of points and the dependence of the results on the limitation of the approximation interval.

\section{REFERENCES}

1. C. A. Hamilton, Rev. Sci. Instrum. 71, 3611 (2000).

2. A. M. Klushin, S. I. Borovitski, C. Weber, et al., in Proceedings of the 3rd European Conference on Applied Superconductivity (EUCAS'97), 1997, Vol. 1, Inst. Phys. Conf. Ser., No. 158, p. 587.

3. A. M. Klushin, W. Prusseit, E. Sodtke, et al., Appl. Phys. Lett. 69, 1634 (1996).

4. S. I. Borovitskiŭ, A. M. Klushin, T. B. Korotina, et al., Pis'ma Zh. Tekh. Fiz. 11, 663 (1985) [Sov. Tech. Phys. Lett. 11, 275 (1985)].

5. B. Jeanneret, A. Rüfenacht, and J. Burroughs, IEEE Trans. Instrum. Meas. 50, 188 (2001).

6. A. M. Klushin, C. Weber, M. Darula, et al., Supercond. Sci. Technol. 11, 609 (1998).

7. R. Behr, H. Schulze, F. Müller, et al., IEEE Trans. Instrum. Meas. 48, 270 (1999).

8. T. J. Witt, IEEE Trans. Instrum. Meas. 50, 445 (2001).

9. K. K. Likharev, Introduction to the Dynamics of Josephson Junctions (Nauka, Moscow, 1985), pp. 177-183.

10. Guide for the Expression of Uncertainty in Measurement (International Organization for Standardization, Switzerland, 1993), Annex H.

11. E. Goldobin, http://www.geocities.com/SiliconValley/Heights/7318/review.htm.

12. Kh. A. Aĭnitdinov, S. I. Borovitskiı̆, V. G. Bykov, et al., Radioizmer. Élektron. KVARTS, No. 1, 5 (1994).

Translated by V. Isaakyan 